\title{
Declínio cognitivo em idosos institucionalizados: revisão de literatura
}

\author{
Cognitive impairment on institucionalized elderly: a literature review
}

Josiane Teresinha Bertoldi', Ana Camila Batista', Samanta Ruzanowsky'

'Faculdade Guilherme Guimbala - Associação Catarinense de Ensino (FGG/ACE), Joinville, SC, Brasil.

Recebido em: novembro 2014 / Aceito em: junho 2015

bertoldi.josi@gmail.com

\section{RESUMO}

O processo de envelhecimento acarreta o comprometimento do sistema nervoso central (SNC), atingido tanto por fatores intrínsecos, quanto extrínsecos. Estas alterações reduzem progressivamente a capacidade intelectual, afetando $5 \%$ das pessoas acima de 65 anos e $20 \%$ acima de 80 anos. A perda da independência e a incapacidade, aliadas à falta de assistência social e de políticas públicas para a população geriátrica, ocasionam a internação do idoso em instituições de longa permanência. A mudança de ambiente e o isolamento social, provocados pela institucionalização, correlacionam-se com a alta prevalência de doenças mentais. Objetivo: investigar, na literatura, a presença de déficit cognitivo em pacientes institucionalizados, avaliados através do Mini-exame do estado mental (MEEM). Método: o estudo refere-se a uma revisão de literatura. A busca de artigos científicos concentrou-se nas bases de dados PUBMED, SCIELO, LILACS, restringindo as publicações entre os anos de 2002 a 2013. Foram selecionados 10 artigos, sendo 09 deles de estudos do tipo quantitativo, descritivo, transversal, e um de revisão literária, que avaliaram ao todo 432 pacientes, na faixa etária entre 60 e 104 anos. Resultados e considerações finais: o prejuízo cognitivo em idosos institucionalizados é elevado, quando comparado a idosos da comunidade. Alguns estudos justificam estas diferenças pelo baixo nível de escolaridade, a idade avançada, o gênero, a falta de atividade física e o isolamento social, sugerindo que a institucionalização é um fator relevante no declínio intelectual. No entanto, destacamos que outras variáveis podem interferir nestes resultados e merecem maior investigação.

Palavras-chave: Institucionalização; Rastreamento Cognitivo; Déficit Cognitivo em Idosos.

\section{ABSTRACT}

The aging process compromises the central nervous system (CNS), reached by both intrinsic and extrinsic factors. These changes progressively reduce the intellectual capacity, affecting 5\% of people over 65 and $20 \%$ over 80 years. The loss of independence and inability coupled with lack of social welfare and public policy for the geriatric population causes the admission of the elderly in nursing homes. Changes in social living and isolation caused by institutionalization are associated with the high prevalence of mental illness. Objective: this research refers to a literature review in order to investigate the presence of cognitive impairment in institutionalized patients assessed by the Mini-Mental State Examination (MMSE). Method: database searches for papers were conducted in PUBMED, SCIELO and LILACS, restricting publications between the years 2002 to 2013. Ten papers were included, nine of then containing quantitative, descriptive, cross-sectional studies, and one literature review comprising 432 patients with ages between 60 and 104 years. Results and closing remarks: results show that cognitive impairment in institutionalized elderly is high when compared to elderly who lives on the community. Some studies justify these differences by the low level of education, advanced age, gender, lack of physical activity and social isolation, suggesting that institutionalization is a relevant factor in the intellectual decline. However, we emphasize that other variables may affect these results and deserve further investigation.

Keywords: Institutionalization; Cognitive Screening; Cognitive Impairment in Elderly. 


\section{INTRODUÇÃO}

O crescimento da população idosa é um processo gradual e inevitável, um fenômeno mundial que acompanha a elevação da qualidade de vida, decorrentes de melhores oportunidades de trabalho, educação, condições sanitárias, alimentares, ambientais de moradia, além do maior acesso aos serviços de saúde. ${ }^{1-3}$ No Brasil, os dados demográficos demonstram que o número de idosos vem crescendo expressivamente e de acordo com a Organização Mundial de Saúde, em 2025 haverá, aproximadamente, 32 milhões de pessoas com mais de 60 anos. ${ }^{4,5}$

O processo de envelhecimento acarreta o comprometimento do sistema nervoso central, atingido, tanto por fatores intrínsecos, quanto extrínsecos (meio ambiente, entre outros). Estas alterações reduzem a capacidade intelectual, afetando as funções de memória, raciocínio lógico, juízo crítico, funções práxicas e gnósicas, orientação espacial, afetividade, personalidade e atitude, fala e outras formas de comunicação. ${ }^{6,7}$

Durante um processo de envelhecimento, aproximadamente $15 \%$ das pessoas desenvolvem incapacidade cognitiva progressiva, em média $5 \%$ das pessoas acima de 65 anos e $20 \%$ acima de 80 anos desenvolvem demência moderada a grave. ${ }^{8,9} \mathrm{~A}$ demência é comum em idosos; representa um grande problema de saúde pública, afeta diferentes funções cognitivas e é considerada a maior causa de incapacidade e mortalidade. ${ }^{9-11}$

A perda da independência e a incapacidade do idoso, aliadas à falta de assistência social e de políticas públicas para a população geriátrica, são fatores que levam a institucionalização do idoso. ${ }^{12}$ Estudos demonstram que a demanda por instituição de longa permanência para idosos (ILPI) no Brasil é crescente..$^{13,14}$

A mudança de ambiente provocada pela institucionalização é uma das situações que desencadeiam a depressão no idoso; apontada, frequentemente, na literatura, como fator de risco para o déficit cognitivo e demência. Esse isolamento social leva à perda da identidade, de liberdade, de autoestima, ao estado de solidão e muitas vezes renegar a própria vida, o que justifica o declínio cognitivo e a alta prevalência de doenças mentais. ${ }^{6,14}$

A investigação das alterações cognitivas, pode ser realizada através de diversos protocolos validados, dentre eles o MEEM (Mini exame do estado mental), que é de fácil e rápida aplicação, e é capaz de avaliar o prejuízo cognitivo. ${ }^{15}$ Tal exame foi desenvolvido por Folstein, ${ }^{16}$ em 1975 e apresenta grande confiabilidade, compõe-se de sete categorias, cada uma delas planejada com o objetivo de avaliar funções cognitivas específicas, são elas: orientação para tempo, orientação para local, registro de três palavras, atenção e cálculo, recordação das três palavras, linguagem e praxia visuo-construtiva. $\mathrm{O}$ escore do MEEM varia de 0 a 30 pontos, sendo que escore abaixo de 24 pontos aponta para diagnóstico de demência. No Brasil, o escore foi classificado para diferentes graus de escolaridade por Bertolucci et al. ${ }^{17}$ em 1995.

O objetivo desta pesquisa foi realizar uma revisão de literatura sobre a presença de déficit cognitivo em pacientes institucionalizados, avaliados através do Mini-exame do estado mental (MEEM).

\section{MÉTODO}

Trata-se de um estudo de revisão literária, de artigos científicos publicados nas bases de dados PUBMED, SCIELO, LILACS. A busca foi conduzida, utilizando as palavras-chave: institucionalização de idosos, avaliação cognitiva em idosos, déficits cognitivos em idosos institucionalizados, rastreamento cognitivo, bem como similares na língua inglesa: elderly institutionalization, elderly cognitive impairment, cognitive function, cognitive tests, cognitive screening. A busca retornou 208 artigos, sendo excluídos os artigos repetidos nas bases de dados nacionais, artigos relacionados com outras patologias do SNC e aqueles que não utilizaram o protocolo MEEM na avaliação. As buscas foram concentradas para publicações de 2002 a 2014.

\section{RESULTADOS E DISCUSSÃO}

Foram selecionados 10 artigos que abordavam a avaliação cognitiva de idosos institucionalizados e utilizavam o protocolo MEEM no rastreamento cognitivo, 09 estudos do tipo quantitativo, descritivo, transversal, e uma revisão de literatura. As pesquisas envolveram ao todo 432 pacientes, na faixa etária entre 60 e 104 anos. Neste sentido, segue abaixo uma tabela contendo os estudos identificados, expondo os principais dados da pesquisa, como a quantidade de participantes, escores alcançados e variáveis dos resultados.

O menor desempenho cognitivo de idosos internados em instituições asilares comparados à idosos que vivem na sociedade, sugerem que a institucionalização pode agravar o declínio cognitivo. ${ }^{11}$ Um estudo, envolvendo 53 idosos de ambos os sexos, na faixa etária de 72,33 $\pm 10,44$ anos, divididos em dois grupos, sendo G1 composto por 22 idosos institucionalizados e o G2 contendo 31 idosos não institucionalizados, avaliados através do MEEM, apontou comprometimento cognitivo severo no primeiro grupo com resultado de 11,73 \pm 6,04, e funções preservadas no grupo 2, com média de 26,39 $\pm 3,2 .{ }^{11}$ Outra pesquisa observou que das 50 pessoas participantes, divididas em dois grupos, 25 idosos institucionalizados e 25 não institucionalizados, o escore do $\mathrm{G} 1$ foi $12,56 \pm 5,034$ e do grupo 2 foi de $24,72 \pm 2,909$, determinando que os idosos que vivem exclusos e longe da família apresentam perda da capacidade cognitiva, justificados pela baixa escolaridade e pelo tempo de institucionalização. ${ }^{18,19}$

O baixo nível de escolaridade e a idade avançada são fatores de risco para o comprometimento cognitivo e déficit funcional. ${ }^{20}$ Cerca de $60 \%$ de 60 idosos, idade $75,0 \pm 11,60$ anos, institucionalizados, avaliados pelo MEEM no município de Jequié-BA, tem alteração cognitiva $^{13}$. O déficit foi ainda maior em idosos internados em IPLI, com faixa etária mais avançada, variando entre 62 e 104 anos. O escore médio obtido foi de 13 pontos, apontando déficit cognitivo moderado em $76,72 \%$ das 115 pessoas avaliadas. ${ }^{10}$ Outro estudo correlacionou a capacidade funcional com a função cognitiva em 30 idosos, com idade média de 78,2 $\pm 4,0$ anos, através da aplicação do MEEM e constatou escore médio total de $18,2 \pm 6,0$ e déficit de mobilidade funcional. ${ }^{9}$ 
Tabela 1 - Avaliação do estado cognitivo: autor, amostra, métodos, variáveis e conclusão.

\begin{tabular}{|c|c|c|c|c|}
\hline Referência & Amostra & Metodologia & Variáveis & Conclusão \\
\hline $\begin{array}{l}\text { TRINDADE A., } \\
\text { et al. }{ }^{11} \\
(2013)\end{array}$ & $\begin{array}{l}\mathrm{n}=53 \\
-22 \text { em ILP } \\
\text { ( } 17 \text { Mulheres e } 5 \text { Homens) } \\
-31 \text { comunidade } \\
\text { ( } 22 \mathrm{M} \text { e } 9 \mathrm{H}) \\
\text { Idade } 72,33 \pm 10,44\end{array}$ & $\begin{array}{l}\text { MEEM } \\
\text { GDR } \\
\text { Lawton }\end{array}$ & $\begin{array}{l}\text { - 54,55\% declarou que nunca estudou } \\
\text { - e nenhum idoso declarou que tenha } \\
\text { estudado por mais de cinco anos. } \\
\text { - Tempo de institucionalização: } \\
\text { não informado. }\end{array}$ & $\begin{array}{l}\text { Os idosos institucionalizados } \\
\text { apresentam menor desempenho } \\
\text { cognitivo com comprometimento das } \\
\text { habilidades funcionais e aumento da } \\
\text { depressão em relação a idosos que } \\
\text { vivem na sociedade e participam de } \\
\text { alguma atividade física. }\end{array}$ \\
\hline $\begin{array}{l}\text { CAIXETA, G.C.S, } \\
\text { et al. }{ }^{19} \\
(2009)\end{array}$ & $\begin{array}{l}\mathrm{n}=50 \\
\mathrm{G} 1-\mathrm{PU}=25 \\
\mathrm{G} 2-\mathrm{VPA}=25 \\
\text { Não informa quantas mul- } \\
\text { heres e quantos homens. } \\
\text { Idade } 72,48 \pm 8,05\end{array}$ & $\begin{array}{l}\text { MEEM } \\
\text { Escala de Equilíbrio de Berg }\end{array}$ & $\begin{array}{l}\text { G1: } 48 \% \text { tinham escolaridade primária } \\
\text { G2: } 80 \% \text { não tinham escolaridade. } \\
\text { Tempo de institucionalização não infor- } \\
\text { mado. }\end{array}$ & $\begin{array}{l}\text { A pesquisa mostrou que os idosos } \\
\text { insti-tucionalizados em ILPI que não } \\
\text { planejam programas de atividades } \\
\text { físicas apresentam menor desem- } \\
\text { penho cognitivo e comprometimento } \\
\text { do equilíbrio funcional em relação aos } \\
\text { idosos que vivem na comunidade e } \\
\text { praticam atividade física. }\end{array}$ \\
\hline
\end{tabular}

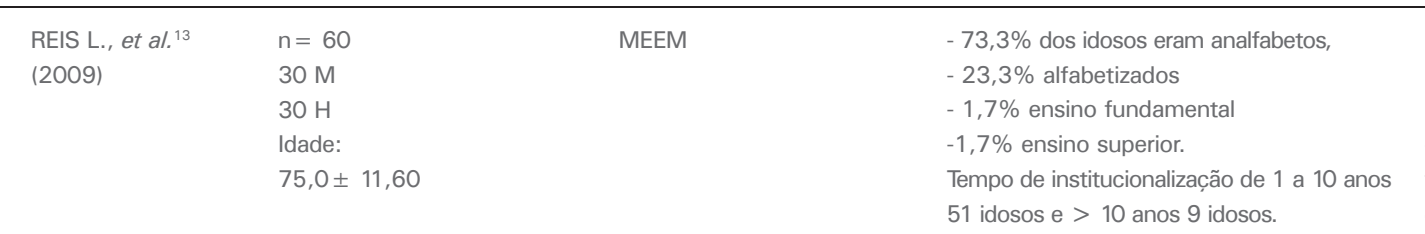

\begin{tabular}{|c|c|c|c|}
\hline $\begin{array}{l}\text { LIRA M, et al. }{ }^{9} \\
(2012)\end{array}$ & $\begin{array}{l}\mathrm{n}=30 \\
22 \mathrm{M} \\
08 \mathrm{H} \\
\text { Idade: } 78,2 \pm 4,0\end{array}$ & $\begin{array}{l}\text { MEEM } \\
\text { TUG-T }\end{array}$ & $\begin{array}{l}\text {-Analfabeto: } 03 \\
\text { - } 1^{\circ} \text { grau incompleto: } 15 \\
-1^{\circ} \text { grau completo :06 } \\
\text { - } 2^{\circ} \text { grau: } 04 \\
\text { - Superior completo:02 } \\
\text { Não informa tempo de internação. }\end{array}$ \\
\hline $\begin{array}{l}\text { SILVA A.O, et al. }{ }^{4} \\
\text { (2011) }\end{array}$ & $\begin{array}{l}\mathrm{n}=10 \\
7 \mathrm{M} \\
3 \mathrm{H} . \\
\text { Idade: } 79,5 \pm 10,7\end{array}$ & $\begin{array}{l}\text { MEEM } \\
\text { GDR } \\
\text { Inventário de Ansiedade } \\
\text { Traço Estado (IDATE) }\end{array}$ & $\begin{array}{l}\text { Dos } 10 \text { idosos participantes, } \\
\text { dois não eram alfabetizados } \\
\text { - Tempo de institucionalização: } 23,1 \\
( \pm 20,7) \text { meses. }\end{array}$ \\
\hline
\end{tabular}

O presente estudo evidenciou que as alterações cognitivas em idosos institucionalizados, assim como o encontrado na literatura, foram mais frequentes em idosos com baixo nível de escolaridade.

Os achados neste estudo revelaram que capacidade funcional e função cognitiva se correlacionam de forma linear. Sendo assim, é possível afirmar que, na população estudada, quanto mais baixa foi a função cognitiva, menor foi a sua capacidade funcional.

Quase metade dos idosos em ILPI avaliados $(40 \%)$ apresentam déficit cognitivo, com risco de desenvolvimento de demência. Mais da metade $(60 \%)$ dos idosos apresentam características de depressão. Os idosos institucionalizados avaliados apresentam tendência a desenvolver ansiedade.

\begin{tabular}{|c|c|c|c|c|}
\hline $\begin{array}{l}\text { BASTOS C.L, } \\
\text { et al. } \\
(2010)\end{array}$ & $\begin{array}{l}\mathrm{n}=20 \\
10 \mathrm{M} \\
10 \mathrm{H} \\
\text { Idade: acima de } 60 \text { anos }\end{array}$ & $\begin{array}{l}\text { WAIS-III / Lista de REY } \\
\text { MEEM } \\
\text { Teste Zülliger e uma entre- } \\
\text { vista visando ao diagnós- } \\
\text { tico diferencial. }\end{array}$ & $\begin{array}{l}\text { Homens: todos possuíam } 3^{\circ} \text { grau de } \\
\text { escolaridade. / Mulheres: } 8 \text { pacientes tinham } \\
\text { menos de } 4 \text { anos de escolaridade e } 2 \\
\text { tinham entre } 8 \text { e } 12 \text { anos de estudo. } \\
\text { Tempo de institucionalização: não informado. }\end{array}$ & $\begin{array}{l}\text { Foi possível verificar que é o nível de } \\
\text { escolaridade que explica as diferen- } \\
\text { ças de desempenho entre homens e } \\
\text { mulheres idosos em testes neuropsi- } \\
\text { cológicos. }\end{array}$ \\
\hline $\begin{array}{l}\text { CONVERSO M.E.R, } \\
\text { et al. }{ }^{10} \\
(2007)\end{array}$ & $\begin{array}{l}\mathrm{n}=115 \\
47 \mathrm{M} \\
68 \mathrm{H} \\
\text { Idade:62 a } 104 \text { anos }\end{array}$ & $\begin{array}{l}\text { MEEM } \\
\text { Índice de Barthel }\end{array}$ & $\begin{array}{l}\text { - 50,43\% analfabetos } \\
\text { - 49,56\% alfabetizados. } \\
\text { Tempo de institucionalização: } \\
\text { não informado. }\end{array}$ & $\begin{array}{l}\text { Alta taxa de idosos com possível } \\
\text { demência }(76,72 \%) \text { e idosos inde- } \\
\text { pendentes funcionalmente }(75,65 \%) \text {, } \\
\text { demonstrando que os aspectos } \\
\text { cognitivos são fatores determinantes } \\
\text { para a institucionalização por parte } \\
\text { dos familiares e/ou responsáveis. }\end{array}$ \\
\hline $\begin{array}{l}\text { FERREIRA, A. P., } \\
\text { et } a{ }^{22} \\
(2014)\end{array}$ & $\begin{array}{l}\mathrm{n}=60 \\
47 \mathrm{M} \\
13 \mathrm{H} \\
\text { Idade } 78,8 \pm 9,5\end{array}$ & $\begin{array}{l}\text { MEEM } \\
\text { Teste de Trilha } \\
\text { CDR }\end{array}$ & $\begin{array}{l}\text { Tempo de institucionalização: } \\
\text { não informado. }\end{array}$ & $\begin{array}{l}\text { Verificou-se que o percentual de } \\
\text { idosos com perda cognitiva por meio } \\
\text { do MEEM foi de } 30 \% \text {, sendo mais } \\
\text { prevalente em mulheres. }\end{array}$ \\
\hline $\begin{array}{l}\text { BORGHI A., } \\
\text { et al. } .^{23} \\
(2009)\end{array}$ & $\begin{array}{l}\mathrm{n}=34 \mathrm{M} \\
\text { Idade } 79,82 \pm 8,23\end{array}$ & MEEM & $\begin{array}{l}\text { - 64,7\% eram idosas com até dois anos } \\
\text { de educação formal } \\
\text { - 29,4\% possuíam entre três e dez anos } \\
\text { de escolaridade, } \\
\text { - 5,9\% declararam mais de dez anos de } \\
\text { escolaridade } \\
\text { - Tempo de institucionalização: } 19 \mathrm{M}<10 \text { anos; } \\
15 \mathrm{M}>10 \text { anos }\end{array}$ & $\begin{array}{l}\text { Elevada prevalência de declínio } \\
\text { cognitivo em mulheres, com baixa } \\
\text { escolaridade, idade avançada e muito } \\
\text { tempo de institucionalização. }\end{array}$ \\
\hline
\end{tabular}


O perfil cognitivo de idosos em ILPI foi caracterizado por possível demência, sinais de depressão e tendência à ansiedade, conforme observado no estudo com 10 participantes, com idade $79 \pm 10,7$ anos, institucionalizados na cidade de Uruguaiana-RS, avaliados pelo MEEM, e com escore médio de 17,7 pontos $\pm 6,2{ }^{4}$ Apesar de $66 \%$ dos idosos avaliados apresentarem déficit cognitivo, não foram encontradas alterações psicopatológicas da personalidade, nem transtornos depressivos significativos demonstrados em outra pesquisa, realizada com 20 participantes institucionalizados, destes, 10 do sexo masculino e 10 do sexo feminino, com idade acima de 60 anos. ${ }^{21}$

A perda cognitiva tem maior prevalência nas mu Iheres. Na avaliação do estado cognitivo de 60 idosos, sendo 47 mulheres (M) e 13 homens $(H)$, a prevalência de déficit foi observada em 36,2\% (M), contra 7,7\% $(\mathrm{H})$, com a pontuação variando de 6 a 30 pontos. ${ }^{22}$ Outra pesquisa envolvendo 34 idosas institucionalizadas, com idade entre 65 e 80 anos, identificou que 17,6\% das mulheres não completaram 13 pontos no MEEM, 38,2\% das idosas apresentaram comprometimento cognitivo moderado, cerca de 35,3\% estavam com declínio leve, e apenas $8,9 \%$ alcançaram mais de 26 pontos. Os escores médios foram menores entre os grupos com idades mais avançadas e entre as analfabetas. ${ }^{23}$

$O$ alto índice de pacientes com demência senil em ILPI foi objeto de uma pesquisa de revisão de literatura que abordou o perfil de dementados e os principais aspectos clínicos envolvidos. Verificou-se que é comum idosos em estágios mais avançados de demência, mais velhos e mais dependentes e, normalmente, contam com pouca ou nenhuma assistência familiar. Em relação aos aspectos clínicos envolvidos na institucionalização, destaca-se a alta prevalência de infecções, desnutrição, incontinência urinária, distúrbios de comportamento e imobilidade. ${ }^{24}$

\section{CONSIDERAÇÕES FINAIS}

As pesquisas apontam que a presença de prejuízo cognitivo em idosos institucionalizados é relevante e sugerem que a internação em ILPI é um fator relevante no declínio das funções mentais. Diversos fatores justificam os achados, dentre eles a idade avançada, a baixa escolaridade, o sexo feminino, a falta de atividades físicas e o isolamento social provocado pela própria institucionalização. No entanto, salienta-se que existem outras variáveis como, por exemplo, os déficits cognitivos pré-existentes, que podem influenciar os resultados e merecem maior investigação.

\section{REFERÊNCIAS}

1. Nordon DG, Guimarães RR, Kozonoe DY, Manchilia VS, Dias Neto VS. Perda cognitiva em idosos. Rev Fac Ciênc Méd Sorocaba 2009; 11(3): 5-8.

2. Gurian $M$, Oliveira $R$, Laprega $M$, Júnior $A$. Rastreamento de idosos não institucionalizados. Rev Bras Geriatr Gerontol 2012; 15(2): 275-283. DOI: http://dx.doi.org/10.1590/ S1809-98232012000200010.

3. Gurian M. Rastreamento cognitivo por instrumento baseado no MEEM em idosos não institucionalizados residentes em Batatais - S.P. Dissertação de Mestrado. 87p., 2002.

4. Silva O, Pereira A, Gonçalves D, Viera A, Medeiros R, Altermann C, Sant`anna L, Piccinni A, Carpes P. Perfil cognitivo de idosos institucionalizados de Uruguaiana - RS. Rev Contexto e Saúde 2011; 10(20) :1185-1190.

5. Maciel A, Araújo L. Fatores associados às alterações na velocidade da marcha e força de preensão manual em idosos institucionalizados. Rev Bras Geriatr Gerontol 2010; 13(2): 179-189. DOI: http://dx.doi.org/10.1590/S180998232010000200003.

6. Fichmam H, Fernandes C, Oliveira M, Caramelli P, Aguair D, Novaes R. Predomínio de comprometimento cognitivo leve disexecutivo em idosos atendidos no ambulatório de geriatria de um hospital público terciário na cidade do Rio de Janeiro. Rev. Neuropsicológica Latino-Americana 2013; 5(2): 31-40. DOI: http://dx.doi.org/10.5579/rnl.2013.131.

7. Mello BLD, Haddad MCL, Dellazora MSG. Avaliação cognitiva de idosos institucionalizados. Acta Scientiarum 2012; 34(1): 95-102. DOI: http://dx.doi.org/10.4025/actascihealthsci. v34i1.7974.

8. Ruwer SL, Rossi AG, Simon LF. Equilíbrio no idoso. Rev Bras Otorrinolaringol 2005; 71(3): 298-303.

9. Lira M, Santos LCCS. Correlação entre função cognitiva e capacidade funcional nos indivíduos com doença de Alzheimer. CCBS Cadernos de Pós Graduação em Distúrbio do Desenvolvimento de São Paulo 2012; 12(2): 36-45.

10. Converso MER, Lartelli I. Caracterização e analise do estado mental e funcional de idosos institucionalizados em instituições publicas de longa permanência. J Bras Psiquiatr 2007; 56(4): 267-272. DOI: http://dx.doi.org/10.1590/ s0047-20852007000400005.

11. Trindade APNT, Barbosa MA, Oliveira FB, Borges APO. Repercussão no declínio cognitivo na capacidade funcional em idosos institucionalizados e não institucionalizados. Fisioter Mov 2013; 26(2): 289-290. DOI: http://dx.doi. org/10.1590/S0103-51502013000200005.

12. Vila CP, Silva MEM, Simas JPN, Guimarães ACA, Parcias SR. Aptidão funcional de nível de atenção em idosos praticantes de exercícios físicos. Rev Bras Geriatr Gerontol 2013; 16(2): 355-364.

13. Reis LA, Torres GV, Araújo CC, Reis LA, Novaes LKN. Rastreamento cognitivo de idosos institucionalizados no município de Jequié - BA. Psicologia em Estudo 2009; 14(2): 295-301.

14. Loreiro APL, Lima AA, Silva RCG, Najjar ECA. Reabilitação cognitiva em idosos institucionalizado: um estudo piloto. Rev Ter Ocup Univ São Paulo 2011; 22(2): 136-144.

15. Freitas MAV, Scheicher ME. Qualidade de vida de idosos institucionalizados. Rev Bras Geriatr Gerontol 2010; 13(3): 395-401.

16. Folstein MF, Folstein SE, Mchugh PR. Mini-Mental State. A pratical method for grading the cognitive state of patients for the clinician. Journal of Psychiatry Research 1975; 12(3): 189-198.

17. Bertolucci PHF, Brucki SMD, Campacci SR, Juliano Y. O miniexame do estado mental em uma população geral: impacto da escolaridade. Arq Neuro-Psiquiatr 1994; 52(1): 81-88.

18. Apostolo J, Rosa A, Castro I, Cardoso D. Cognitive Stimulation in elderly people. Revista Portuguesa de Enfermagem de Saúde Mental 2013; 10:24-28.

19. Caixeta GCS, Ferreira A. Desempenho cognitivo e equilíbrio funcional em idosos. Rev. Neurociênc 2009; 17(3): 202-208. 
20. Fagundes SD, Silva MT, Thaes MFRS, Pereira MG. Prevalence of among elderly Brazilians: a systematic review. São Paulo Med. J 2011; 129(1): 46-50. DOI: http://dx.doi.org/10.1590/ S1516-31802011000100009.

21. Coelho CLM, Bastos CL, Camara FP, Fernandes JL. A influência do gênero e da escolaridade no diagnóstico de demência. Estudos de Psicologia Campinas 2010; 27(4): 449-456. DOI: http://dx.doi.org/10.1590/S0103-166X2010000400003.

22. Lenart $\mathrm{MH}$, Michel T, Wachholz PA, Borghi AS, Seima MD.
O desempenho de idosas institucionalizadas no mini exame do estado mental. Acta Paul Enferm 2009; 22(5): 638-644.

23. Ferreira LS, Pinho MSP, Pereira MWM, Ferreira AP. Perfi cognitivo de idosos residentes em Instituições de longa Permanência de Brasília-DF. Rev Bras Enferm 2014; 67: $247-$ 251. DOI: http://dx.doi.org/10.5935/0034-7167.20140033.

24. Gorzoni ML, Pires SL. Aspecto clinico da demência senil em instituições asilares. Rev Psiq Clín 2006; 33(1): 18-23. DOI: http://dx.doi.org/10.1590/S0101-60832006000100003. 